\title{
New semiconductor materials for magnetoelectronics at room temperature
}

\author{
S K KAMILLA and S BASU* \\ Institute of Technical Education and Research, Bhubaneswar 751 030, India \\ *Materials Science Centre, Indian Institute of Technology, Kharagpur 721 302, India
}

\begin{abstract}
Most of the semiconductor materials are diamagnetic by nature and therefore cannot take active part in the operation of the magneto electronic devices. In order to enable them to be useful for such devices a recent effort has been made to develop diluted magnetic semiconductors (DMS) in which small quantity of magnetic ion is introduced into normal semiconductors. The first known such DMS are II-VI and III-V semiconductors diluted with magnetic ions like Mn, Fe, Co, Ni, etc. Most of these DMS exhibit very high electron and hole mobility and thus useful for high speed electronic devices. The recent DMS materials reported are (CdMn)Te, (GaMn)As, (GaMn)Sb, ZnMn(or Co)O, TiMn(or Co)O etc. They have been produced as thin films by MBE and other methods. This paper will discuss the details of the growth and properties of the DMS materials and some of their applications.
\end{abstract}

Keywords. Diluted magnetic semiconductor; II-VI and III-V alloys; oxides.

\section{Introduction}

The past three decades have witnessed the rapid advancement of solid state electronics, including first the replacement of discrete circuit elements and finally the integration of many circuit elements into one semiconductor chip i.e. integrated circuit devices. The only fundamental discrete circuit elements that have been left behind are those involving magnetic materials (Prinz 1990). Semiconductors and magnetic materials are two very important materials in the current electronic industries (Tanaka 1998). In this discussion we are combining both the properties and form new materials in which the performance of devices can be improved. Those materials are known as 'diluted magnetic semiconductors' (DMS). Thus the concept of 'magnetoelectronics' has come up i.e. the electronic chips consisting of DMS materials.

Diluted magnetic semiconductors (DMS) are expected to play an important role in interdisciplinary materials science and future electronics because charge and spin degrees of freedom accommodated into a single material exhibits interesting magnetic, magneto-optical, magnetoelectronic and other properties (Mizokawa et al 2002).

Within the next few years, it is expected that magnetoelectronic chips will be used in quantum computers. An inherent advantage of magnetoelectronics over electronics is the fact that magnet tend to stay magnetized for long. Hence this arises interest in industries to replace the semiconductor-based components of computer with magnetic ones, starting from RAM. The new magnetic RAM

\footnotetext{
*Author for correspondence
}

will retain data even when the computer is turned off. And most important advantage will be eliminating the time consuming process of 'booting up' information from hard drive to processor like a TV set, all the information would be there. One challenge in realizing magnetic RAM involves addressing individual memory elements, flipping their spins up or down to yield the zero and ones of binary computer logic.

DMS are semiconductors in which a fraction of the component ions are replaced by those of transition metals or rare earths (figure 1). Most importantly, state of magnetization changes the electronic properties and vice versa through the spin exchange interaction between local magnetic moments and carriers.

In order to be practical, magnetoelectronics will need to use semiconductors that maintain their magnetic properties at room temperature. This is a challenge because most magnetic semiconductors lose their magnetic properties at temperatures well below room temperature, and would require expensive and impractical refrigeration in order to work in actual computer (Chambers 2001).

\section{Materials and methods}

Preparations of these materials are mainly done by (i) bulk crystal growth e.g. Bridgman method and (ii) thin film growth e.g. (a) molecular beam epitaxy (MBE), (b) pulse laser depositions (PLD) and (c) chemical vapour depositions (CVD).

The bulk growth of DMS materials are limited by solid solubility of magnetic ions in the lattice of the semiconductor crystals. This is because of the fact that 
the equilibrium growth is controlled by phase diagrams.

In contrast thin film growth is non-equilibrium in nature, therefore, by controlling the growth temperature a large concentration of magnetic ions can be introduced in the crystal lattice of the semiconductor.

One of the approaches for the integration of semiconductors and magnetic materials is the epitaxial growth. The term epitaxy is applied to processes used to grow a thin film in such a way that the layer grown has the same lattice structure as substrate.

\subsection{Alloy of II-VI semiconductors with magnetic materials}

Diluted magnetic semiconductors (DMS) are compound of alloy semiconductors containing a large fraction of magnetic ions $\left(\mathrm{Mn}^{+2}, \mathrm{Cr}^{+2}, \mathrm{Fe}^{+2}, \mathrm{Co}^{+2}\right)$ and are studied mainly on II-VI based materials such as CdTe and ZnSe etc. This is because such +2 magnetic ions are easily incorporated into the host II-VI crystals by replacing group II cations. In such II-VI based DMS such as (CdMn)Se, magneto-optic properties were extensively studied, and optical isolators were recently fabricated using their large Faraday effect (Tanaka 1998). Although this phenomenon makes these DMS relatively easy to prepare in bulk form as well as thin epitaxial layers, IIVI based DMS are difficult to dope to create $p$ - and $n$-type, which makes the material less attractive for applications.

The magnetic interaction in II-VI DMSs is dominated by the anti-ferromagnetic exchange among the Mn spins, which results in the paramagnetic, antiferromagnetic, or spins glass behaviour of the material. It was not possible until very recently to make a II-VI DMS ferromagnetic at low temperature $(<2 \mathrm{~K})$.

Recently, the II-VI compound semiconductors $\mathrm{ZnO}$, GaN have attracted revival attention since it was found that high quality epitaxial thin film display excitonic ultraviolet laser action at room temperature (Matsukura et al 1998; Ohtmo et al 1998). In addition, the energy gap of this compound can be extended up to $\sim 4 \mathrm{eV}$ by synthesizing alloy compounds of $\mathrm{Mg}_{x} \mathrm{Zn}_{1-x} \mathrm{O}$. Heavy elec- tron doping (>10 $0^{21} \mathrm{~cm}^{-3}$ ) was readily achieved in contrast to the other II-VI compound semiconductors. Furthermore, the thermal equilibrium solubility of magnetic materials (such as $\mathrm{Mn}$ ) is larger than $10 \mathrm{~mol} \%$ and the electron mass is as large as $\sim 0.3 m_{\mathrm{e}}$, where $m_{\mathrm{e}}$ is free electron mass. Therefore, the amount of injected spin and carriers into the film can be very large. When we take into account the Ruderman-Kittel-Kasuya-Yosida (RKKY) interaction, the above mentioned factors of oxide DMS (i.e. $\mathrm{Zn}_{1-x} \mathrm{Mn}_{x} \mathrm{O}$ ) favour strong correlation between spin and carriers (Matsukura et al 1998).

\subsection{Alloys of III-V semiconductors with ferromagnetic properties}

An approach compatible with the semiconductors used in present day electronics is to make nonmagnetic III-V semiconductors magnetic, and even ferromagnetic, by introducing a high concentration of magnetic ions. The III-V semiconductors such as GaAs are already in use in a wide variety of electronic equipment in the form of electronic and optoelectronics devices, including cellular phones (microwave transistors), compact disks (semiconductor lasers), and in many other applications. Therefore, the introduction of magnetic III-V semiconductors opens up the possibility of using a variety of magnetic phenomena not present in conventional nonmagnetic III-V semiconductors used in the optical and electronics devices already established (Ohno 1998).

The major obstacle in making III-V semiconductors magnetic has been the low solubility of magnetic elements (such as Mn) in the compounds. Because the magnetic effects are roughly proportional to the concentration of the magnetic ions, one would not expect a major change in properties with limited solubility of magnetic impurities, of the order of $10^{18} \mathrm{~cm}^{-3}$ or less. Using molecular beam epitaxy (MBE), a thin film growth technique in vacuum that allows one to work far from equilibrium, made a breakthrough. When a high concentration of magnetic elements is introduced in excess of the solubility limit, formation of the second phase occurs if conditions are near equilibrium (Ohno 1998).

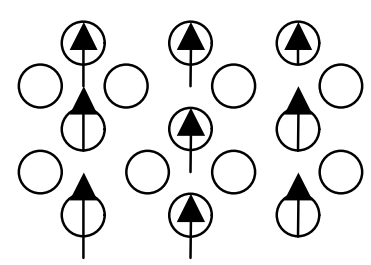

(A)

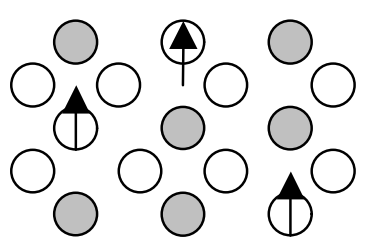

(B)

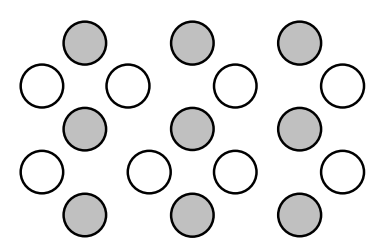

(C)

Figure 1. Three types of semiconductors: (A) a magnetic semiconductor, in which a periodic array of magnetic element is present, (B) a diluted magnetic semiconductor, an alloy between nonmagnetic semiconductor and magnetic element and (C) a non-magnetic semiconductor, which contains no magnetic ions. 
Magnetic III-V semiconductors show ferromagnetism, whose curie temperature, $T_{\mathrm{c}}$, is relatively high as predicted theoretically only when the carrier concentration is effectively raised.

However, the highest curie temperature, $T_{\mathrm{c}}$, obtained to date is about $110 \mathrm{~K}$ in $\mathrm{Ga}_{1-x} \mathrm{Mn}_{x} \mathrm{As}$ (Matsukura et al 1998).

\section{Outline of our proposed research}

So far bulk crystals of (GaMn)Sb, (GaFe)Sb DMS have been grown by vertical Bridgman method in our laboratory and have been characterized for optical, electrical and magnetic properties (Adhikari and Basu 1994; Karar and Basu 1999). These materials showed curie temperature below room temperature i.e. $300 \mathrm{~K}$. So our present purpose is to develop oxide DMS materials like $\mathrm{ZnO}, \mathrm{TiO}_{2}$ doped with the first transition metals like $\mathrm{Mn}, \mathrm{Co}, \mathrm{Fe}, \mathrm{Ni}$ etc. Most of the work on the DMS materials are based on $\mathrm{Mn}$ as the magnetic component. So our plan is to develop $\mathrm{Co}, \mathrm{Fe}, \mathrm{Ni}$ based thin film in order to manifest the direct ferromagnetic DMS behaviour for application devices.

The purpose of this work is to develop the knowledge as to the design and fabrication of advanced magnetic semiconductor materials based on II-V or III-V semiconductors.

\section{Conclusion}

$\mathrm{ZnO}, \mathrm{TiO}_{2}$ doped with 3-d transition metals $\mathrm{Co}, \mathrm{Ni}, \mathrm{Fe}$ etc are promising DMS materials showing ferromagnetism with $T_{\mathrm{c}}$ above room temperature. The large band gap may open up transparent ferromagnetic materials in oxide DMS for application in the visible region.

\section{References}

Adhikari T and Basu S 1994 Jpn. J. Appl. Phys. 334581

Chambers S A 2001 Paper presented at spintronic workshop, Washington D.C.

Karar N and Basu S 1999 Mater. Sci. and Eng. B56 21

Matsukura F, Ohno H, Shen A and Sugawara Y 1998 Phys. Rev. B57 R2037

Mizokawa T, Namba T, Fujimori A, Fukumura T and Kawasaki M 2002 Phys. Rev. $\mathbf{B 6 5} 085209$

Ohno H 1998 Science 281951

Ohtmo A, Kawasaki M, Koida T, Masubuchi K, Koinuma H, Sakurai Y, Yoshida Y and Segawa Y 1998 Appl. Phys. Lett. 722466

Prinz Gary A 1990 Science $\mathbf{2 5 0} 1092$

Tanaka M 1998 J. Vac. Sci. Technol. B16 2267 